\title{
Inference of Nonlinear State-space Models for Sandwich-Type Lateral Flow Immunoassay Using Extended Kalman Filtering
}

\author{
Nianyin Zeng, Zidong Wang, Yurong Li, Min Du* and Xiaohui Liu
}

\begin{abstract}
In this paper, a mathematical model for sandwichtype lateral flow immunoassay is developed via short available time series. A nonlinear dynamic stochastic model is considered that consists of the biochemical reaction system equations and the observation equation. After specifying the model structure, we apply the extend Kalman filter (EKF) algorithm for identifying both the states and parameters of the nonlinear state-space model. It is shown that the EKF algorithm can accurately identify the parameters and also predict the system states in the nonlinear dynamic stochastic model through an iterative procedure by using a small number of observations. The identified mathematical model provides a powerful tool for testing the system hypotheses and also inspecting the effects from various design parameters in a both rapid and inexpensive way. Furthermore, by means of the established model, the dynamic changes of the concentration of antigens and antibodies can be predicted, thereby making it possible for us to analyze, optimize and design the properties of lateral flow immunoassay devices.
\end{abstract}

Index Terms-Lateral flow immunoassay; Extended Kalman filtering; Gold immunochromatographic strip; Mathematical model; Parameter estimation.

\section{INTRODUCTION}

In the past few years, the rapid immunochromatographic test strip, also called lateral flow immunoassay(LFIA), has been under especially intensive investigations because of its advantages such as ease of use, short analysis time, low cost, high sensitivity, good specificity, satisfactory stability when applied to a wide variety of point-of-care (POC) tests [20], [22], [33]. Owing to these attractive properties, the lateral flow immunoassay has been widely used in many fields including clinical diagnostics [29], food safety testing [14],

Copyright (c) 2010 IEEE. Personal use of this material is permitted. However, permission to use this material for any other purposes must be obtained from the IEEE by sending an email to pubs-permissions@ieee.org.

This work was supported in part by the International Science and Technology Cooperation Project of China under Grant 2009DFA32050, Natural Science Foundation of Fujian Province of China under Grants 2009J01280 and 2009J01281. Asterisk indices corresponding author.

${ }^{*} \mathrm{M}$. Du is with the College of Physical and Information Engineering, Fuzhou University, Fuzhou 350002, P. R. China, and also with the Fujian Key Laboratory of Medical Instrumentation and Pharmaceutical Technology, Fuzhou 350002, P. R. China. Email addresses: dm_dj90@163.com.

N. Zeng and Y. Li are with the College of Electrical Engineering and Automation, Fuzhou University, Fuzhou 350002, P. R. China, and also with the Fujian Key Laboratory of Medical Instrumentation and Pharmaceutical Technology, Fuzhou 350002, P. R. China. Email addresses: nianyin.zeng@gmail.com (N. Zeng), liyurong@fzu.edu.cn (Y. Li).

Z. Wang and X. Liu are with the Department of Information Systems and Computing, Brunel University, Uxbridge, Middlesex, UB8 3PH, United Kingdom. Email address: Zidong.Wang@brunel.ac.uk (Z. Wang), Xiaohui.Liu@brunel.ac.uk (X. Liu). environmental health and safety [39], agriculture [38], as well as some emerging areas such as molecular diagnostics and theranostics [11].

The lateral flow immunoassay, which utilizes the specific interaction between antigens and antibodies, consists of a porous membrane or strip that is often made out of nitrocellulose. The primary antibodies are immobilized within a defined detection zone (test line) on the membrane. The secondary antibodies are conjugated with reporter particles such as colloidal gold, carbon black, fluorescent, or paramagnetic monodisperse latex particle [1]. In this paper, we focus on the sandwich format of gold immunochromatographic strip where the reporter particle uses the colloidal gold nanoparticles. Although the lateral flow immunoassay technology is widely used in a variety of areas, the format suffers from certain shortcomings such as test-totest reproducibility challenges for quantitative analysis and the hook effect happened when the high concentration of analyte exist in the sample. Therefore, most immunochromatographic assays can only give qualitative or semi-quantitative results observed directly by naked eyes at present [2] which, in turn, significantly limit the applications of these assays.

Recent research has been going mainly towards two directions: one is the material selection and the improvement of biochemical property of strips, see e.g. [19], [23], [35], and the other is the development of quantitative instruments, see e.g. [4]-[7], [13], [24], [25]. In order to produce highsensitivity results with low constant of variance (CV) strip and enable the quantification, there is an urgent need for improved materials, assay technology, reader technology and manufacturing processes. There is also a growing research interest on a more multidisciplinary approach to lateral flow development [33]. In this sense, it is of great importance to establish a mathematical model that allows us to predict kinetic characteristics and also test the effects of various design parameters in a both rapid and inexpensive way. In addition to providing insights into device operation, such a model could also enable us to optimize device performance [30]. Unfortunately, up to now, little research has been done on the general modeling issues for lateral flow immunoassay systems except the work [30], [31] relying on the convection diffusion reaction equations and the COMSOL software for simulation.

In order to gain an insight into the performance of the lateral flow immunoassay system, one needs to actually focus on the modeling issue of biochemical reaction networks between the antigens and the antibodies. In principle, the lateral flow immunoassay model can be described by a nonlinear state- 
space system that is characterized by the state equations and observation equations. The system state equations describe the dynamics of the concentration distribution subject to stochastic disturbances, and the system measurements are determined in terms of an observation equation containing measurement noises. After specifying the model structure, we need to find a way to solve the joint parameter and state estimation problem for nonlinear systems with stochastic noises. Nevertheless, in practice, the lateral flow immunoassay system itself exhibits several distinguishing features that add to the difficulties in its modeling: 1) the biochemical reaction between antigens and antibodies typically finishes in at most ten minutes, and therefore we are only able to acquire a small number of experiment data (i.e., short time-series); 2) the measurement is usually concerned with a combination of certain variables and many other system variables are not measureable; and 3) the dynamic model for the lateral flow immunoassay system is essentially nonlinear, and the system parameters and the system states have to be estimated simultaneously. In general, there have been three popular algorithms for the estimation problem of nonlinear systems, namely, extended Kalman filtering [3], [34], [36], unscented Kalman filtering (UKF) [32] and sequential Monte Carlo method [12], [26].

The traditional Kalman filter has been successfully used in linear models [8]-[10] because of its versatility and effectiveness. The EKF approach linearizes the nonlinear model by Taylor expansion and then uses the traditional Kalman filter for the linearized model. The EKF algorithm utilized in estimation problems is quite convenient as we only need to calculate the mean and covariance values of the system states. Moreover, the EKF is known as an effective online (recursive) estimator for process variables, which can be suitable for identifying large number of parameters using a short time series [3]. On the other hand, the other approaches for the parameter estimation problem in nonlinear models, such as the unscented Kalman filtering (UKF) and sequential Monte Carlo method, would require a sufficiently large number of data for the statistical inference. With hope to address the listed challenges for modeling nonlinear lateral flow immunoassay system, the extended Kalman filtering (EKF) approach stands out as an appropriate candidate since it is capable of handling the joint parameter and state estimation problem via short time series data.

In this paper, we aim to infer the nonlinear state-space model for the sandwich-type lateral flow immunoassay using extended Kalman filtering approach through available short time-series. The model is characterized by the system state equation and the system measurement equation. The identified mathematical model enables us to test the system hypotheses conveniently and also inspect the effects brought from various design parameters. By utilizing the established model, we are capable of predicting the dynamic changes of the concentration of antigens and antibodies, and this paves the way for analyzing, optimizing and designing the behaviors of lateral flow immunoassay devices. The main contribution of this paper is mainly twofold. 1) The EKF algorithm is applied to jointly estimate the system parameters, actual concentration distribution of states, the system noise and measurement noise in the nonlinear model of lateral flow immunoassay. Note that the EKF algorithm is an online estimation algorithm that can solve the estimation problem through iterative procedure by using a small number of observations. 2) Real-time experimental data are obtained to evaluate the model identified by the EKF, and it is shown that the model fits the data very well. In particular, the hook effect phenomenon typically exhibited in lateral flow immunoassay can be clearly demonstrated by the established model.

The rest of this paper is organized as follows. In Section II, the lateral flow immunoassay system is introduced and the nonlinear state-space model is proposed for the lateral flow immunoassay system. In Section III, the EKF approach to parameter identification is described. The results of parameter identification and state estimation by the EKF method are discussed in Section IV and the model performance is also demonstrated. Finally, concluding remarks are given in Section V.

\section{The MOdel FOR LATERAL Flow IMMUNOASSAY}

\section{A. Lateral Flow Immunoassay}

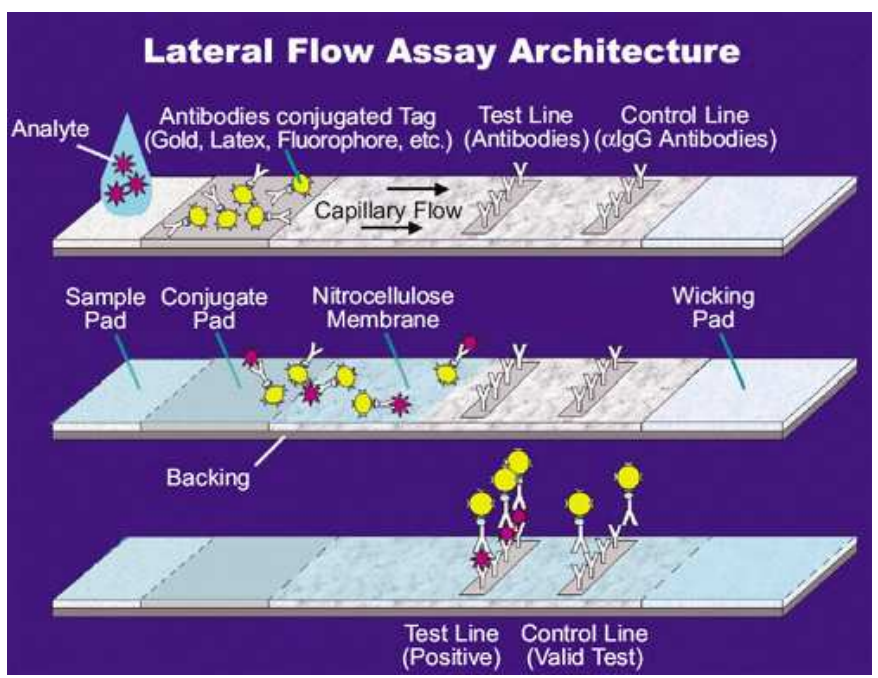

Fig. 1. Lateral flow immunoassay architecture.

A typical configuration of lateral flow immunoassay, as shown in Fig. 1 [16], consists of a variety of materials such as sample pad, nitrocellulose membrane, conjugate pad and wicking pad. With the presence of an antigen in the sample, a sandwich-type assay is formed between the secondary antibody-immobilized gold nanoparticle immunocomplex and the primary antibody immobilized on the membrane. After the antigen-antibody reaction, the red color caused by the accumulation of gold nanoparticle at that location would appear on the membrane [1], [33]. The color intensity of the red test line (signal intensity), which relates directly to the concentration of the target protein in the standard or spiked samples, is assessed visually or by a reader system for quantitative analysis [35].

In general, the biochemical reactions of the lateral flow immunoassay signal pathway can be summarized as follows [30]: 
This article has been accepted for publication in a future issue of this journal, but has not been fully edited. Content may change prior to final publication.

1) Assume that the sample contains various target analytes $A_{i}$. When the sample migrates through the conjugate pad, the analytes interact with the particulate color particle conjugate $P$ to form particle-analyte complexes $P A_{i}$

$$
A_{i}+P \underset{k_{2}}{\stackrel{k_{1}}{\rightleftharpoons}} P A_{i}
$$

2) The free analytes in the sample and the particle-analyte complexes both migrate into the membrane by the capillary action. Free analytes of type $i\left(A_{i}\right)$ and particleanalyte complexes $P A_{i}$ interact with the immobilized ligands of type $i\left(R_{i}\right)$ to form the complexes,

$$
\begin{gathered}
A_{i}+R_{i} \underset{k_{4}}{\stackrel{k_{3}}{\rightleftharpoons}} R A_{i} \\
P A_{i}+R_{i} \underset{k_{6}}{\stackrel{k_{5}}{\rightleftharpoons}} R P A_{i}
\end{gathered}
$$

3) Additionally, unbound particulate conjugate $P$ may bind to the complex $R A_{i}$ to form the complex $R P A_{i}$,

$$
P+R A_{i} \underset{k_{8}}{\stackrel{k_{7}}{\rightleftharpoons}} R P A_{i}
$$

In the above, we assume that the first-order reversible interactions occur without consideration of the control line. In this paper, for simplicity, we only consider a single target analyte in the sample, and therefore we drop the subscript $i$ from the next section.

\section{B. Lateral Flow Immunoassay Model}

Let $x_{1}, x_{2}, x_{3}, x_{4}, x_{5}$ and $x_{6}$ be the concentration of $A, P, P A, R, R A$ and $R P A$, respectively. For demonstration purpose, it is assumed that there is no time-delay between the biochemical reactions (1)-(4). The rates of the reactions are defined as follows:

$$
\begin{aligned}
& v_{1}=k_{1} x_{1} x_{2}-k_{2} x_{3} \\
& v_{2}=k_{3} x_{1} x_{4}-k_{4} x_{5} \\
& v_{3}=k_{5} x_{3} x_{4}-k_{6} x_{6} \\
& v_{4}=k_{7} x_{2} x_{5}-k_{8} x_{6}
\end{aligned}
$$

where $k_{1}, k_{3}, k_{5}, k_{7}$ and $k_{2}, k_{4}, k_{6}, k_{8}$ are the association and dissociation rate constants, respectively. The stoichiometrix for the biochemical reaction of the lateral flow immunoassay is given by

$$
S=\left[\begin{array}{cccc}
-1 & -1 & 0 & 0 \\
-1 & 0 & 0 & -1 \\
1 & 0 & -1 & 0 \\
0 & -1 & -1 & 0 \\
0 & 1 & 0 & -1 \\
0 & 0 & 1 & 1
\end{array}\right]
$$

Let $x=\left[x_{1}, x_{2}, \ldots, x_{6}\right]^{T}$ and $V=\left[v_{1}, v_{2}, \ldots, v_{4}\right]^{T}$. The differential equation for the biochemical reactions of the lateral flow immunoassay is given as follows [17], [34]

$$
\frac{d x(t)}{d t}=S V(x)
$$

It should be pointed out that the variables $x_{1}, x_{2}, x_{3}, x_{4}, x_{5}$ and $x_{6}$ are not measurable/observable. The only observed signal that can be detected with a reader system is the test line's intensity, which is typically either the color intensity or the phosphor emission intensity or fluorescent [30]. The signal would be proportional to the concentration of particleanalyte complexes $P A$ and the complex $R P A$. In this case, the observation equation is obtained as follows

$$
y=k_{9}\left(x_{3}+x_{6}\right) \text {. }
$$

The most general form of the nonlinear model for the dynamics of biochemical networks is defined by dynamic mass balance equations or kinetic models [34], where the system consists of a pair of equations as follows

$$
\begin{aligned}
& d x(t)=f(x(t), t) d t+G(t) d \beta(t) \\
& d y(t)=g(x(t), t) d t+L(t) d \eta(t)
\end{aligned}
$$

where $x(t)$ is the vector of state variables which are concentrations of antibodies, antigens or complex material; $y(t)$ is the measurement process; $f(x(t), t)=S V(x(t))$ with $S$ being a stoichiometric matrix that describes the biochemical transformation in a biochemical network and $V(x(t))$ being the vector of reaction rates (usually the vector of nonlinear function of the state) [34]; $G(t)$ and $L(t)$ are arbitrary timevarying matrices independent of $x(t)$ and $y(t) ; g(x(t), t)$ is the measurement model function; and $\beta(t)$ and $\eta(t)$ are independent Brownian motions with diagonal diffusion matrices $Q(t)$ and $R(t)$, respectively. For the convenience of engineering applications, such a model can also be modified in terms of the white noises $w(t)=d \beta(t) / d t$ and $v(t)=d \eta(t) / d t$ as follows [18]:

$$
\begin{aligned}
\frac{d x}{d t} & =S V(x(t))+G(t) w(t) \\
z(t) & =g(x(t))+L(t) v(t)
\end{aligned}
$$

where the Gaussian white noises $w(t)$ and $v(t)$ are uncorrelated and independent for all $t$.

In practice, when modeling biochemical networks from observed data (time series), discrete-time models play a more crucial role than their continuous-time counterparts in today's digital world. In order to obtain the nonlinear model for lateral flow immunoassay biochemical networks from discretely obtained measurements, it is usually essential to formulate the discrete-time analogue as follows [34]:

$$
\begin{aligned}
x(k+1) & =x(k)+S V(x(k))+w(k) \\
z(k) & =g(x(k))+v(k)
\end{aligned}
$$

To facilitate the parameter estimation, in this paper, let us use $\theta=\left[k_{1}, k_{2}, \ldots, k_{9}\right]^{T}$ to denote the parameters to be estimated, which are the association and dissociation rate constants in the vector $V(x(k))$. Therefore, we can rewrite the model (15)-(16) in the following more compact form:

$$
\begin{aligned}
x(k+1) & =f(x(k), \theta)+w(k) \\
z(k) & =g(x(k), \theta)+v(k)
\end{aligned}
$$

where $x(k)$ is the vector of state variables at the time point $k, f(.,$.$) is a nonlinear function with \theta$ being a parameter 
vector to be identified. $w(k)$ and $v(k)$ denote the zero-mean uncorrelated Gaussian noises with covariance matrices $Q_{k}$ and $R_{k}$, respectively. $z(k)$ is the measurement data from experiments at the time point $k$.

It is clear from (17)-(18) that what we need to do is to identify the parameter vector $\theta$ for the purpose of establishing the lateral flow immunoassay model. The main aim of this paper is to estimate the parameters of the model (17)-(18) via the EKF method from the possibly small number of the measured data.

\section{Joint PARAmeter And State Estimation By the EXTENDED KALMAN FilTER}

In this section, for the convenience of the readers, we introduce the EKF approach to parameter identification, see e.g. [3], [27], [36] for more details. The Kalman filter is the optimum state estimator for a linear system with the assumptions as described. If the system is nonlinear, then we may use a linearization process at every time step to approximate the nonlinear system with a linear time varying (LTV) system. This LTV system is then used in the Kalman filter, resulting in an extended Kalman filter (EKF) on the true nonlinear system. Note that although EKF is not necessarily optimal, it often works very well. Discussions on the convergence of EKF can be found in [15], [21] and the references therein.

Consider the following nonlinear system

$$
\begin{aligned}
x(k+1) & =f(x(k))+w(k) \\
y(k) & =g(x(k))+v(k)
\end{aligned}
$$

where $k$ is a non-negative integer, $x(k) \in \mathbb{R}^{n}$ is the system state vector, $y(k) \in \mathbb{R}^{r}$ is the observation vector, $w(k)$ and $v(k)$ are the system noise and the measurement noise, respectively. $w(k)$ and $v(k)$ are zero-mean white Gaussian stochastic processes with covariance matrices $Q_{k}$ and $R_{k}$, respectively. Here, $f: \mathbb{R}^{n} \rightarrow \mathbb{R}^{n}$ is a nonlinear state transition function and $g: \mathbb{R}^{n} \rightarrow \mathbb{R}^{r}$ is a nonlinear measurement function.

The extended Kalman filter is implemented by the following consecutive steps:

1) Consider the last filtered state estimate $\hat{x}(k \mid k)$

2) Linearize the system dynamics (19) around $\hat{x}(k \mid k)$

3) Apply the prediction step of the Kalman filter to the linearized system dynamics just obtained, yielding $\hat{x}(k+$ $1 \mid k)$ and $P(k+1 \mid k)$

4) Linearize the observation equation (20) around $\hat{x}(k \mid k)$

5) Apply the filtering or update cycle of the Kalman filter to the linearized observation dynamics, yielding $\hat{x}(k+$ $1 \mid k+1)$ and $P(k+1 \mid k+1)$

Let

$$
\begin{aligned}
\hat{A}(k) & =\left.\frac{\partial f(x(k))}{\partial x(k)}\right|_{x(k)=\hat{x}(k \mid k)} \\
\hat{C}(k) & =\left.\frac{\partial g(x(k))}{\partial x(k)}\right|_{x(k)=\hat{x}(k \mid k-1)}
\end{aligned}
$$

Assume that $x(0) \sim \mathcal{N}\left(x_{0}, P_{x_{0}}\right), w(k) \sim \mathcal{N}\left(0, Q_{k}\right), v(k) \sim$ $\mathcal{N}\left(0, R_{k}\right)$ with $R_{k}>0$, and that $\{w(k)\}$ and $\{v(k)\}$ are white noise processes uncorrelated with $x(0)$ and with each other.
Then, the Extended Kalman filter algorithm can be stated below:

\section{Initialization}

For $k=0$, set

$$
\begin{aligned}
& \hat{x}(0 \mid 0)=E[x(0)]=x_{0}, \\
& P(0 \mid 0)=E\left[\left(x(0)-x_{0}\right)\left(x(0)-x_{0}\right)^{T}\right]=P_{x_{0}} .
\end{aligned}
$$

For $k=1,2,3, \ldots$ compute

Time update ('Predict')

State estimate time update: $\hat{x}(k \mid k-1)=f(\hat{x}(k-1 \mid k-1))$

Error covariance time update: $P(k \mid k-1)=\hat{A}(k-1) P(k-$ $1 \mid k-1) \hat{A}(k-1)^{T}+Q_{k-1}$

Measurement update ('Correct')

Compute the Kalman gain matrix: $K_{k}=P(k \mid k-$ 1) $\hat{C}^{T}(k)\left[\hat{C}(k) P(k \mid k-1) C(k)^{T}+R_{k}\right]^{-1}$

Update the estimate with measurement $y(k): \hat{x}(k \mid k)=$ $\hat{x}(k \mid k-1)+K_{k}[y(k)-g(\hat{x}(k \mid k-1))]$

Error covariance measurement update: $P(k \mid k)=(I-$ $\left.K_{k} \hat{C}(k)\right) P(k \mid k-1)$.

In addition, in order to improve the precision of state estimation and also reduce the possible biases, there is a need to properly quantify the parameters $Q$ and $R$ in the EKF algorithm. To tackle this issue, we use the innovationbased adaptive estimation (IAE) approach [40], where the covariance matrices $Q(k)$ and $R(k)$ are estimated and then updated iteratively according to the following equations:

$$
\begin{aligned}
R(k) & =C_{v k}+\hat{C}(k) P(k \mid k) \hat{C}(k)^{T} \\
Q(k) & =K_{k} C_{v k} K_{k}^{T}
\end{aligned}
$$

where $C_{v k}$ is the innovation covariance matrix computed through averaging the innovation sequence $s(k)$ inside a moving estimation window of size $N$,

$$
\begin{aligned}
C_{v k} & =\frac{1}{N} \sum_{i=k-N+1}^{k} s(k) s(k)^{T} \\
s(k) & =y(k)-g(\hat{x}(k \mid k-1))
\end{aligned}
$$

Based on the above equations, the appropriate values of $Q$ and $R$ can be determined at each iteration.

Remark 1: Extended Kalman filter is a very practical method in identification of nonlinear systems. Augmenting the unknown parameters to the state vector makes it possible to use EKF for parameter identification too.

\section{Results Using THE EKF APPROACH AND DISCUSSION}

In this paper, we take human chorionic gonadotropin (hCG) as a target analyte. The CCD-based image acquisition system [25] is exploited to generate the short time series shown in Fig. 2. The upper part of Fig. 2 shows the gold immunochromatographic strip's nitrocellulose membrane pixel intensity inverse the sample flow direction. The images are acquired when the sample passes through the nitrocellulose membrane that consists of 45 equally spaced time points (from 0 to 11 minutes), 45 images as a time series. We choose $z=\left[\max _{\text {pixel }}\left(255-I_{\text {pixel }}\right)-P_{\text {base }}\right] / 10$ as the observed value shown in the lower part of Fig. 2, where $I_{\text {pixel }}$ is the pixel 

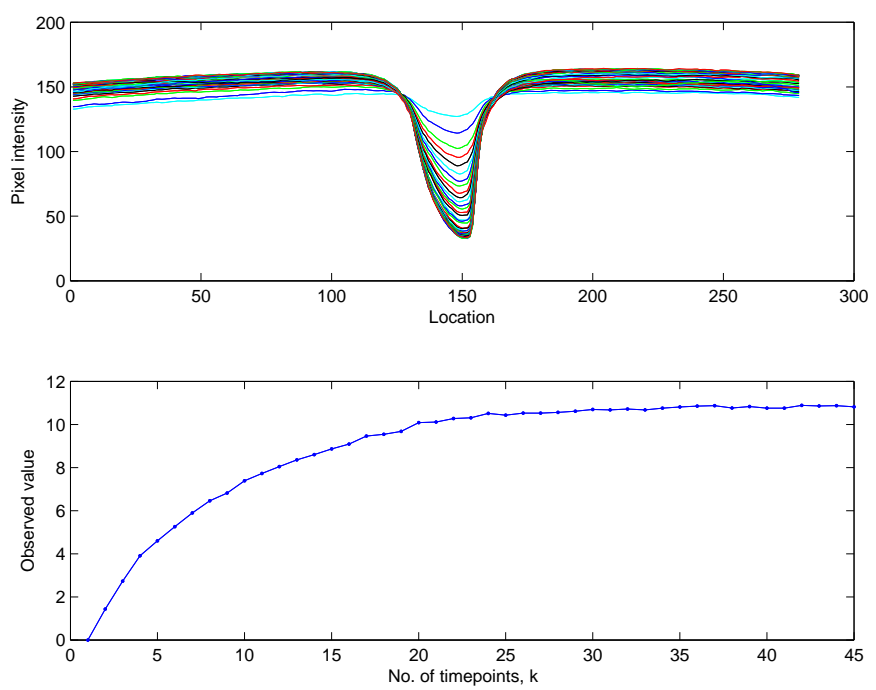

Fig. 2. Upper part: The strip's nitrocellulose membrane pixel intensity inverse the sample flow direction; Lower part: The observed value of lateral flow immunoassay biochemical reaction signal

intensity and $P_{\text {base }}$ equals to $\max _{\text {pixel }}\left(255-I_{\text {pixel }}\right)$ of the first out of the 45 images.

Take

$$
x_{0}=[5,6.5,0,13,0,0]^{T}
$$

and

$k_{0}=[0.03,0.0001,0.01,0.0001,0.04,0.0001,0.04,0.0001,2.2]^{T}$

as the initial values of the state variables and parameters, respectively. Then, we can estimate parameters and state variables based on the EKF algorithm. Both the identified parameters and state variables are shown in Fig. 3 and Fig. 4, which are expressed in the form of time series. The time series for error covariances is also obtained, simultaneously, which is depicted in Fig. 5 and Fig. 6 . In Fig. 7 shows the time series for the noise variance $Q_{k}$ and $R_{k}$.

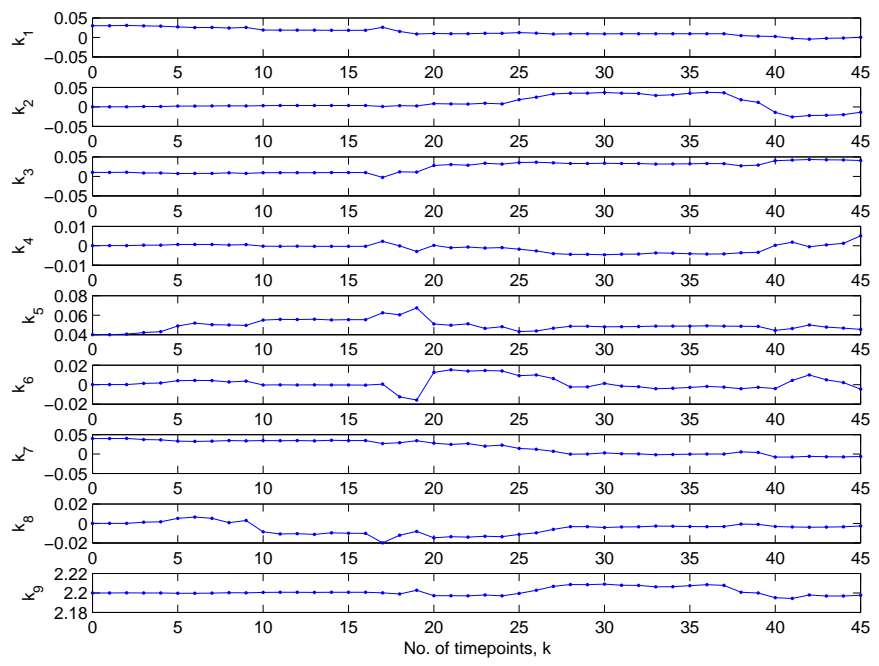

Fig. 3. The estimated time series of parameters $k_{1}, k_{2}, k_{3}, k_{4}, k_{5}$, $k_{6}, k_{7}, k_{8}, k_{9}$.

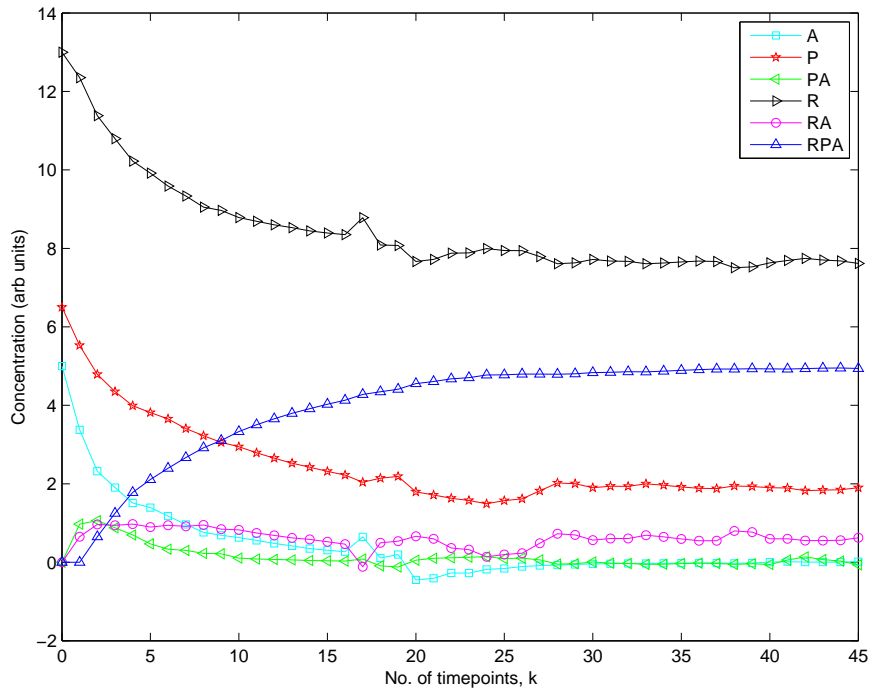

Fig. 4. The estimated time series of states $A, P, P A, R, R A, R P A$.
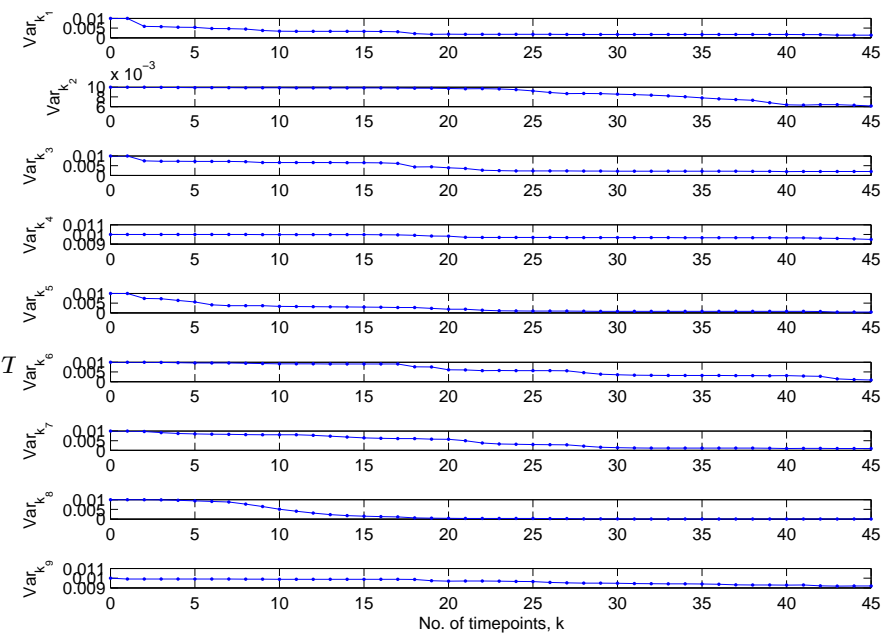

Fig. 5. The variances of estimated time series of parameters $k_{1}, k_{2}$, $k_{3}, k_{4}, k_{5}, k_{6}, k_{7}, k_{8}, k_{9}$.

It can be seen from Fig. 4 that the target analytes $A$, color particle conjugate $P$ and immobilized ligands $R$ are decreasing as the time goes. The particle-analyte complexes $P A$ and the complex $R A$ increase in the first instance because of the biochemical reactions (1) and (2), but later decrease because of the biochemical reactions (3) and (4). Therefore, the complex $R P A$ increases with the time going on and eventually reaches the equilibrium state. It can now be concluded that the model identified by the EKF algorithm does make the practical sense. Also from Fig. 5 and Fig. 6, we can see that the estimation covariances are small, which means that our model fits the data very well.

In order to further evaluate the model identified by the EKF method, we have done some experiments by changing the concentration of the target anylyte. Fig. 8 shows the observed value predicted by the EKF method and observed value from the real experiments. Furthermore, to evaluate the model quality in a quantitative way, let us introduce the following 


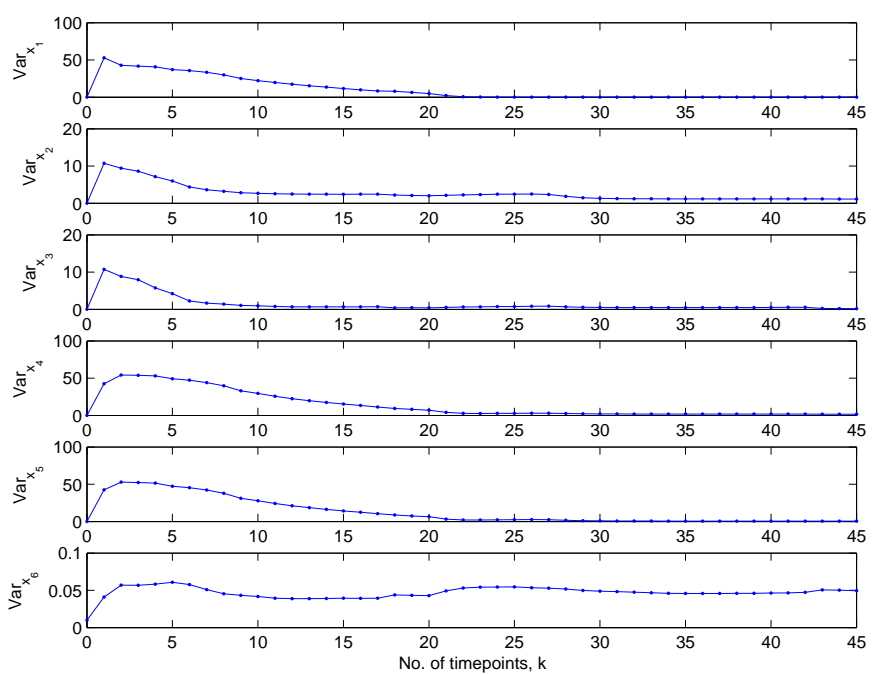

Fig. 6. The variances estimated time series of states $A, P, P A, R$, $R A, R P A$.

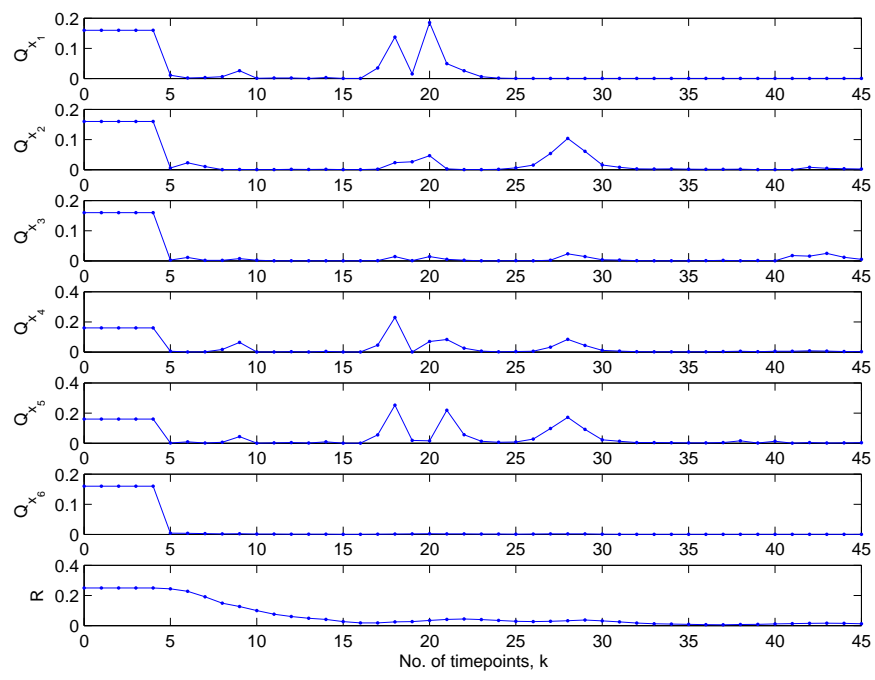

Fig. 7. The noise variances estimated time series of $Q_{k}$ and $R_{k}$.

criterion for the modeling errors (error ratio in percentage) between the actual and the model predicted data [28], [37]:

$$
\text { Error ratio }=100 \times \frac{1}{l} \sum_{c=1}^{l}\left[\sqrt{\frac{\sum_{k=1}^{s}\left(y_{c k}-\hat{y}_{c k}\right)^{2}}{\sum_{k=1}^{s}\left(y_{c k}\right)^{2}}}\right] \%
$$

where $l$ is the number of observations (dimension) involved in the modeling ( $l=1$ in this paper); $s$ is the number of observations (length), and $y_{c k}$ is the actual value for $c$ th observation at the $k$ th time point. The results are given in Table I. Given the fact that there are only 45 time points (images), the model quality is satisfactory.

Next, let us examine if the established model could reveal biological significance. The hook effect is a well-known phenomenon in lateral flow immunoassay when the target analyte is detected to exceed the amount of colored labeling conjugate or the ligands immobile on the capture zone. The cause for such a phenomenon is that the analyte binds both to the colored

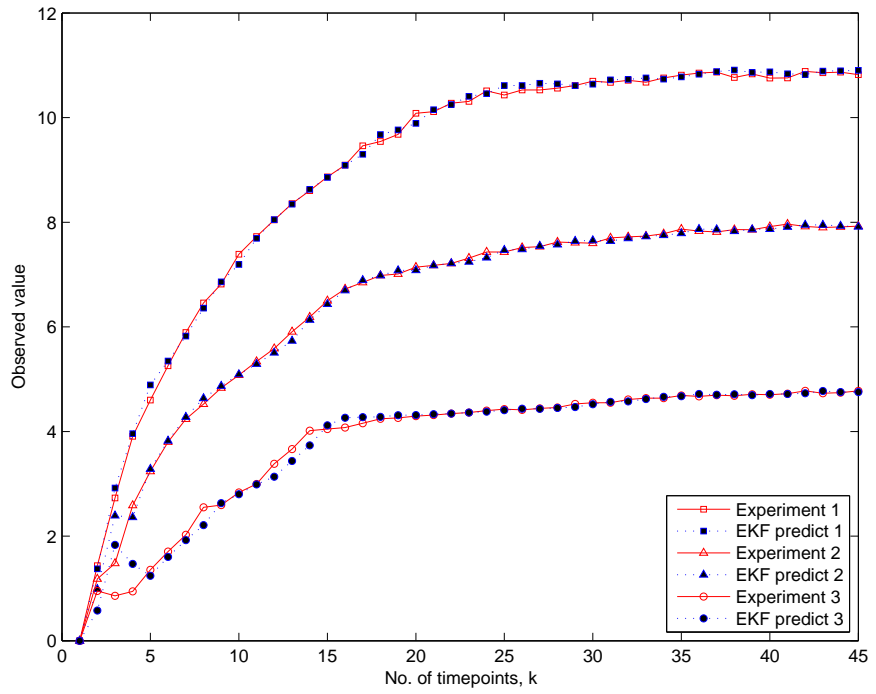

Fig. 8. The observed value predicted by EKF method and observed value from the experiment.

TABLE I

QUANTITATIVE MODEL EVALUATION FOR TIME SERIES WITH DIFFERENT CONCENTRATIONS

\begin{tabular}{|c|c|c|c|}
\hline \hline Experiment & 1 & 2 & 3 \\
\hline Error ratio & $1.05 \%$ & $2.27 \%$ & $5 \%$ \\
\hline \hline
\end{tabular}

labeling conjugate and to the ligands, and also blocks many of the particle-analyte complexes from binding with the ligands [30]. In theory, the identified model by the EKF method should confirm the occurrence of such a phenomenon. Fig. 9 shows the concentration of the complex $R P A$ as a function of the target analyte concentration. When the target analyte concentration is less than the colored labeling conjugate or the ligands, the immunoassay can provide accurate results and the dose-response curve shows a positive slope. The slope will become negative when the concentration of analyte exceeds the ligands [33]. To this end, the expected hook effect has been successfully displayed by the identified model.

Remark 2: When it comes to the quantitative interpretation for the lateral flow immunoassay, best time-points (images) should be obtained by the reader system. For different manufacturers, different materials such as nitrocellulose membrane are used and therefore the criterion for choosing the best time-points is the biochemical reaction. Concerning the above strips' performance, we have chosen to capture the images 6 minutes later after the sample is added in the strip in order to obtain reasonable results. From Fig. 9, we can observed that, despite different eigenvalues, the curve is in qualitative agreement with the experiment results reported in [25] experiment results when the target analyte concentration is less than the colored labeling conjugate or the ligands, and this again shows the validity of the proposed modeling approach. 


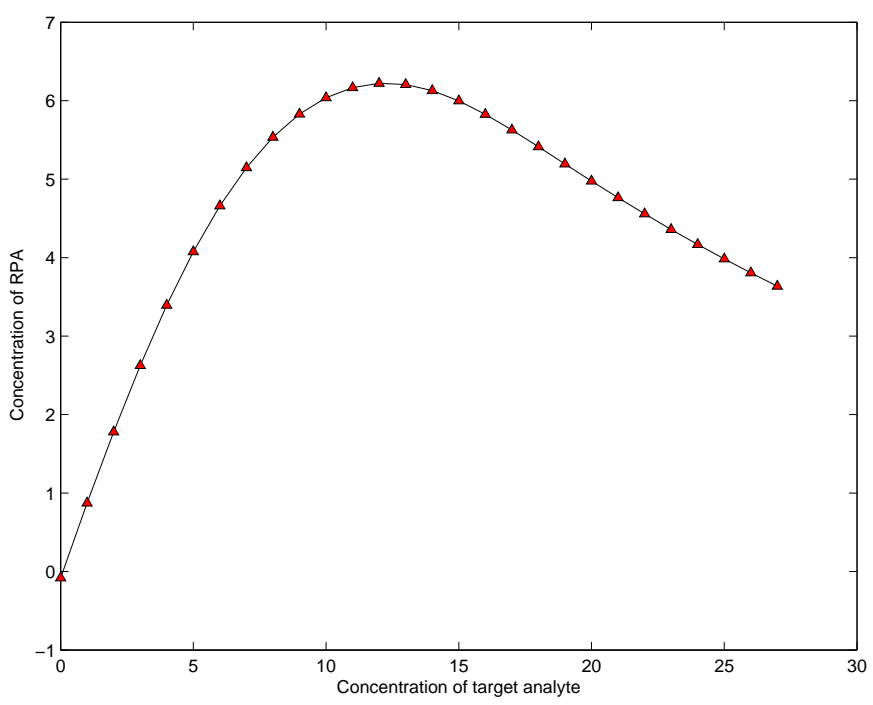

Fig. 9. The concentration of RPA as a function of the target analyte concentration.

\section{CONCLUSiOnS AND Future WORK}

In this paper, we have dealt with three important issues for sandwich-type lateral flow immunoassay. The first one is the identification of nonlinear dynamic stochastic model consisting of system and measurement equations. The second issue is the EKF algorithm applied to jointly estimate the system states and parameters for the lateral flow immunoassay via short time-series data. The third issue is to verify the identified model by using experimental data. It has been shown that the established model fits the data very well and therefore offers a powerful means for testing the system hypotheses and for predicting the dynamic changes of the concentration of antigens and antibodies.

In the near future, we will continue to investigate the modeling issue of the lateral flow immunoassay where the control line is included and the time-delays between the biochemical reactions are taken into account. Furthermore, the variations in membrane properties, batch to batch variability of the goldantibody conjugates and other factors lead to the variability observed from strip to strip at the same concentration, so these factors could be considered as input variables in the statespace model in the next step. Although the EKF algorithm is an efficient estimator because of its recursive nature, this algorithm might result in infeasible estimates because it does not take into account the physical constraints on the estimated states. Therefore, the EKF algorithm with estimation constraints should be considered, and the moving-horizon estimation (MHE) approach appears to be good candidate for incorporating the estimation constraints. We are also getting in touch with biologists and manufacturers to gain further insight into our main results.

\section{REFERENCES}

[1] C. An, T. Yoshiki, G. Lee, Y. Okada, Evaluation of a rapid qualitative prostate specific antigen assay, the One Step PSA ${ }^{T M}$ test, Cancer Lett, vol. 162 , no. 2 , pp. 135-139, 2001
[2] L. Chuang, J. Hwang, H. Chang, F. Chang, and SH. Jong, Rapid and simple quantitative measurement of a-fetoprotein by combining immunochromatographic strip test and artificial neural network image analysis system, Clinica Chimica Acta, vol. 348, pp. 87-93, 2004.

[3] A. Corigliano and S. Mariani, Parameter identification in explicit structural dynamics: performance of the extended Kalman filter, Computer Methods in Applied Mechanics and Engineering, vol. 193, pp. 38073835, 2004.

[4] M. Du, Z. Fang, H. Yu, R. Lu, Y. Zong and Y. Tao, Development of an immunochromatographic assay system for the quantitative determination of alpha-fetoprotein, The Journal of Near-Patient Testing and Technology, vol. 2, no. 1, pp. 14-19, 2003.

[5] M. Du, Z. Fang and H. Fei, Application of photoelectric sensor to quantitative determination of immunochro-matographic assay strip, Chinese Journal of Scientific Instrument, vol. 36, no. 7, pp. 671-673, 2005.

[6] K. Faulstich, R. Gruler, M. Eberhard and K. Haberstroh, Developing rapid mobile POC systems. Part 1:Devices and applications for lateralflow immunodiagnostics, IVD Technology, vol. 13, no. 6, pp. 47-53, 2007.

[7] K. Faulstich, R. Gruler, M. Eberhard and K. Haberstroh, Developing rapid mobile POC systems. Part 2: Nucleic acid based testing platforms, IVD Technology, vol. 13, no. 7, 2007.

[8] H. Gao, X. Meng, and T. Chen, A Parameter-Dependent Approach to Robust $H_{\infty}$ Filtering for Time-Delay Systems, IEEE Trans. Automatic Control, vol. 53, no. 10, pp. 2420-2425, 2008.

[9] H. Gao X. Meng, and T. Chen, A new design of robust $H_{2}$ filters for uncertain systems, Systems and Control Letters, vol. 57, no. 7, pp. 585593, 2008.

[10] Z. Gao and S. X. Ding, State and disturbance estimator for time-delay systems with application to fault estimation and signal compensation, IEEE Transactions on Signal Processing, vol. 55, no. 12, pp. 5541-5551, 2007.

[11] J. Gillespie, G. Gannot, M. Tangrea, M. Ahram, C. Best, V. Bichsel, E. Petricoin, M. Emmert-Buck and R. Chuaqui, Molecular profiling of cancer, Toxicol. Pathol, vol. 32, pp. 67-71, 2004.

[12] X. Hu, T. B. Schon, L. Ljung, A Basic Convergence Result for Particle Filtering, IEEE Trans Signal Processing, vol. 56, no. 4, pp. 1337-1348, 2008.

[13] L. Huang, Y. Zhang, Ch. Xie, J. Qu, H. Huang, and X. Wang, Research of reflectance photometer based on optical absorption, International Journal for Light and Electron Optics, vol. 121, no. 19, pp. 1725-1728, 2010.

[14] S. Huang, H. Wei and Y. Lee, One-step immunochro-matographic assay for the detection of Staphylococcus aureus, Food Control, vol. 18, no. 8 , pp. 893-897, 2007.

[15] S. Huang and D. Dissanayake, Convergence and consistency analysis for extended Kalman filter based SLAM, IEEE Trans. Robotics, vol. 23, no. 5, pp. 1036-1049, 2007.

[16] http://commons.wikimedia.org/wiki/File:Lateral_Flow_Assay.jpg

[17] N. Jamshidi, ВØ. Palsson, Formulating genome-scale kinetic models in the post-genome era, Molecular Systems Biology, vol. 4, no. 171, 2008.

[18] A. Jazwinski, Stochastic processes and filtering theory, New York: Academic, 1970.

[19] J. Kaur, K. Singh, R. Boro, K. Thampi, M. Raje and G. Varshney, Immunochromatographic dipstick assay format using gold nanoparticles labeled protein-hapten conjugate for the detection of atrazine, Environmental Science and Technology, vol. 41, no. 14, pp. 5028-5036, 2007.

[20] A. Kolosova, S. Saeger, L. Sibanda, R. Verheijen and C. Peteghem, Development of a colloidal gold-based lateral-flow immunoassay for the rapid simultaneous detection of zearalenone and deoxynivalenol, Anal. Bioanal. Chem, vol. 389, pp. 2103-2107, 2007.

[21] A.J. Krener, The Convergence of the Extended Kalman Filter, IEEE Directions in Mathematical Systems Theory and Optimization, pp. 173182,2003

[22] E. Laderman, E. Whitworth, E. Dumaual, M. Jones, A. Hudak, W. Hogrefe, J. Carney and J. Groen, Rapid, sensitive, and specific lateral-flow immunochromatographic point-of-care device for detection of herpes simplex virus type 2-specific immunoglobulin $\mathrm{G}$ antibodies in serum and whole blood, Clin. Vaccine Immunol, vol. 5, pp. 159-163, 2008.

[23] D. Li, S. Wei, H. Yang, Y. Li and A. Deng, A sensitive immunochromatographic assay using colloidal gold-antibody probe for rapid detection of pharmaceutical indomethacin in water samples, Biosensors and Bioelectronics, vol. 24, no. 7, pp. 2277-2280, 2009.

[24] J. Li, A. Ouellette, L. Giovangrandi, D. Cooper, A. Ricco and G. Kovacs, Optical scanner for immunoassays with up-converting phosphorescent 
labels, IEEE Trans. Biomedical Engineering, vol. 55, no. 5, pp. 15601571,2008

[25] Y. Li, N. Zeng and M. Du, Study on the methodology of quantitative gold immunochromatographic strip assay, Proc. of the 2010 International Workshop on Intelligent Systems and Application, pp. 182-185, 2010.

[26] L. Lang, W. Chen, BR. Bakshi, PK. Goel and S. Ungarala, Bayesian estimation via sequential Monte Carlo sampling-Constrained dynamic systems, Automatica, vol. 3, pp. 1615-1622, 2007.

[27] F. Lewis, L. Xie and D. Popa, Optimal and robust estimation, CRC press, 2007.

[28] L. Ljung, System Identification: Theory for the User, 2nd ed. Upper Saddle River, NJ: Prentice-Hall, 1999.

[29] R. Lundblad and P. Wagner, The potential of proteomics in developing diagnostics, IVD Technology, vol. 3, pp. 20-22, 2005.

[30] S. Qian and H. Haim, A mathematical model of lateral flow bioreactions applied to sandwich assays, Analytical Biochemistry, vol. 322, no. 1 , pp. 89-98, 2003.

[31] S. Qian and H. Haim, Analysis of lateral flow biodetectors: competitive format, Analytical Biochemistry, vol. 326, no. 2, pp. 211-224, 2004.

[32] M. Quach, N. Brunel and F. d'Alché-Buc, Estimating parameters and hidden variables in non-linear state-space models based on ODEs for biological networks inference, Bioinformatics, vol. 23, no. 23, pp. 32093216, 2007.

[33] C. Raphael and Y. Harley, Lateral flow immunoassay, Humana Press, 2008.

[34] X. Sun, L. Jin and M. Xiong, Extended Kalman filter for estimation of parameters in nonlinear state-space models of biochemical networks, PLoS ONE, vol. 3, no. 11, e3758, 2008.

[35] R. Tanaka, T. Yuhi, N. Nagatani, T. Endo, K. Kerman and Y. Takamura, A novel enhancement assay for immunochromatographic test strips using gold nanoparticles, Anal Bioanal Chem, vol. 385, no. 8, pp. 1414-1420, 2006.

[36] Z. Wang, X. Liu, Y. Liu, J. Liang and V. Vinciotti, An extended Kalman filtering approach to modeling nonlinear dynamic gene regulatory networks via short gene expression time series, IEEE/ACM Trans. Computional Biology and Bioformatics, vol. 6, no. 3, pp. 410-419, 2009.

[37] Z. Wang, F. Yang, D. W. C. Ho, S. Swift, A. Tucker and X. Liu, Stochastic dynamic modeling of short gene expression time series data, IEEE Trans. NanoBioscience, vol. 7, no. 1, pp. 44-55, 2008.

[38] G. Zhang, X. Wang, A. Zhi, Y. Bao, Y. Yang, M. Qu, J. Luo, Q. Li, J. Guo, Z. Wang, J. Yang, G. Xing, S. Chai, T. Shi and Q. Liu, Development of a lateral flow immunoassay strip for screening of sulfamonomethoxine residues, Food Addit Contam Part A, vol. 25, no. 4 pp. 413-423, 2008.

[39] J. Zhu, W. Chen, Y. Lu and G. Cheng, Development of an immunochromatographic assay for the rapid detection of bromoxynil in water, Environmental Pollution, vol. 156, no. 1, pp. 136-142, 2008.

[40] A. Mohamed, K. Schwarz, Adaptive Kalman Filtering for INS/GPS, Journal of Geodesy, vol. 73, no. 4, pp. 193-203, 1999.

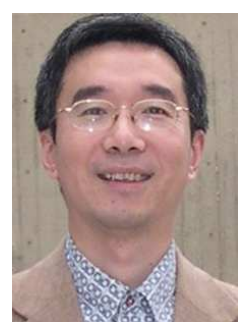

Zidong Wang was born in Jiangsu, China, in 1966. He received his B.Sc. degree in Mathematics in 1986 from Suzhou University, Suzhou, China, the M.Sc. degree in Applied Mathematics in 1990 and the Ph.D. degree in Electrical and Computer Engineering in 1994, both from Nanjing University of Science and Technology, Nanjing, China.

Dr. Wang is now a Professor of Dynamical Systems and Computing at Brunel University in the UK. His research interests include dynamical systems, signal processing, bioinformatics, control theory and applications. He has published more than 120 papers in refereed international journals.

Dr. Wang is currently serving as an Associate Editor for 12 international journals including IEEE Transactions on Automatic Control, IEEE Transactions on Neural Networks, IEEE Transactions on Signal Processing, IEEE Transactions on Systems, Man, and Cybernetics - Part C and IEEE Transactions on Control Systems Technology.

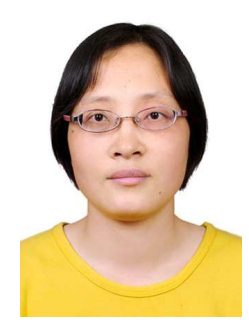

Yurong Li was born in Fujian Province, China, in 1973. She received her master degree in Industry Automation and $\mathrm{PhD}$ in Control Theory and Control Engineering from Zhejiang University, Zhejiang, Chian in 1997 and 2001, respectively.

Now she is an assistant professor at Fuzhou University. And since 2007, she is the member of Fujian Key Laboratory of Medical Instrumentation \& Pharmaceutical Technology. Her research interests include biomedical instrument and intelligent information processing.

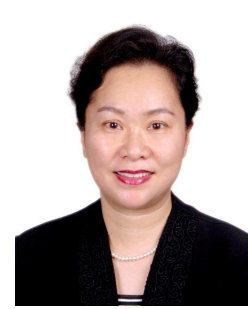

Min Du was born in Fujian Province, China, in 1955. She received her $\mathrm{PhD}$ in Electrical Engineering from Fuzhou University, Fuzhou, China, in 2005.

Now she is a professor and doctorial supervisor at Fuzhou University. And since 2007, she is the associate director of Fujian Key Laboratory of Medical Instrumentation \& Pharmaceutical Technology. Her research interests include smart instrument and photoelectrical system.

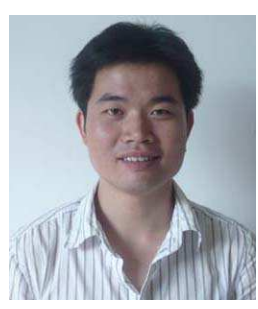

Nianyin Zeng was born in Fujian Province, China, in 1986. He received the B.S. degree in Electrical Engineering and Automation from Fuzhou University, Fuzhou, China, in 2008, where he is currently working toward the Ph.D. degree in Electrical Engineering. His current research interests include systems biology using control methods, computational biology, and bioinformatics.

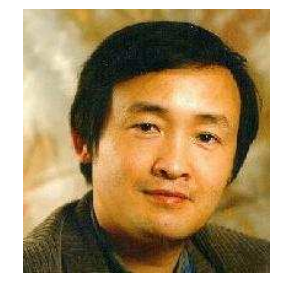

Xiaohui Liu received the B.Eng. degree in computing from Hohai University, Nanjing, China, in 1982 and the Ph.D. degree in computer science from Heriot-Watt University, Edinburg, U.K., in 1988

$\mathrm{He}$ is currently a Professor of Computing at Brunel University. He leads the Intelligent Data Analysis (IDA) Group, performing interdisciplinary research involving artificial intelligence, dynamic systems, image and signal processing, and statistics, particularly for applications in biology, engineering and medicine. Professor Liu serves on editorial boards of four computing journals, founded the biennial international conference series on IDA in 1995, and has given numerous invited talks in bioinformatics, data mining and statistics conferences. 